\title{
TOPOLOGICAL INTERSECTION THEOREMS
}

\author{
JÜRGEN KINDLER
}

(Communicated by Andrew M. Bruckner)

\begin{abstract}
Let $\left\{C_{x}: x \in X\right\}$ be a family of subsets of some set $Y$. A purely topological condition is given that is both necessary and sufficient for $\bigcap\left\{C_{x}: x \in X\right\}$ to be nonvoid. Applications to minimax theorems are sketched.
\end{abstract}

\section{Main Results}

Let $Y$ be a nonvoid set and $\left\{C_{x}: x \in X\right\}$ a family of nonvoid subsets of $Y$ ( $X$ a nonvoid index set). Let $C_{y}^{*}:=\left\{x \in X: y \notin C_{x}\right\}, y \in Y$, denote the system of conjugate sets and set $\mathscr{E}(X):=\{A \subset X: A$ finite nonvoid $\}$. We shall prove the following two theorems:

Theorem 1. The following are equivalent:

(a) $\cap\left\{C_{x}: x \in X\right\} \neq \varnothing$.

(b) There exist topologies on $X$ and $Y$ such that

(o) $Y$ is compact,

(i) every set $C_{x}, x \in X$, is closed,

(ii) for every closed $F \subset Y$ the subset $\bigcap\left\{C_{y}^{*}: y \in F\right\}$ is open,

(iii) every subset $\bigcap\left\{C_{x}: x \in A\right\}, A \in \mathscr{E}(X)$, is connected or empty,

(iv) every subset $\bigcap\left\{C_{y}^{*}: y \in B\right\}, B \subset Y$, is connected or empty.

Here subsets of topological spaces are endowed with the relative topology, and we set $\bigcap\left\{C_{y}^{*}: y \in \varnothing\right\}=X$.

Theorem 2. The following are equivalent:

(a) $\bigcap\left\{C_{x}: x \in A\right\} \neq \varnothing$ for all $A \in \mathscr{E}(X)$, i.e., the sets $C_{x}, x \in X$, have the finite intersection property.

(b) There exist topologies on $X$ and $Y$ such that

(i) every set $C_{x}, x \in X$, is open,

(ii) for every open $G \subset Y$ the subset $\bigcap\left\{C_{y}^{*}: y \in G\right\}$ is closed,

(iii) every subset $\bigcap\left\{C_{x}: x \in A\right\}, A \in \mathscr{E}(X)$, is connected or empty,

(iv) every subset $\bigcap\left\{C_{y}^{*}: y \in B\right\}, B \subset Y$, is connected or empty.

Received by the editors July 11, 1991.

1991 Mathematics Subject Classification. Primary 49J35, 54C60; Secondary 54D05.

Key words and phrases. Intersecting sets, connectedness, upper semicontinuous correspondences, minimax.

(C) 1993 American Mathematical Society $0002-9939 / 93 \$ 1.00+\$ .25$ per page 
Our proof of these two dual theorems is entirely elementary. It owes some inspiration to the study of minimax theorems (compare also the examples below). The source of the idea dates back to 1959 when $\mathrm{Wu}$ pointed out [18] that the only property of convex sets that is actually needed in the proof of minimax theorems is their connectedness. Until now, many authors have used and developed further Wu's method (cf. Tuy [17], Joó [6], Stachó [16], Komornik [12], Geraghty-Lin [3], Komiya [10, 11], Kindler-Trost [8], and Horvath [5] just to mention a few).

\section{CORRESPONDENCES WITH CONSTANT SELECTOR}

It is convenient to reformulate our intersection problem in terms of correspondences. In the following, let $X$ and $Y$ be nonvoid sets and $\Phi$ a correspondence from $X$ to $Y$, i.e., a mapping $\Phi: X \rightarrow \mathscr{P}(Y):=\{B \subset Y: B \neq \varnothing\}$. A mapping $f: X \rightarrow Y$ is a selector for $\Phi$ iff $f(x) \in \Phi(x)$ for all $x \in X$.

Main problem. When does the correspondence $\Phi$ possess a constant selector?

As usual, $\operatorname{Gr} \Phi=\{(x, y) \in X \times Y: y \in \Phi(x)\}$ is the graph of $\Phi$, and $\Phi^{*}: \Phi^{*}(y)=\{x \in X: y \notin \Phi(x)\}, y \in Y$, is the dual of $\Phi$. Recall that a correspondence $\Phi$ from a topological space $X$ to a topological space $Y$ is called upper (lower) semicontinuous iff for every open (closed) $H \subset Y$ the set $\{x \in X: \Phi(x) \subset H\}$ is open (closed).

Remark 1. Let $X$ be a topological space, $Y$ a nonvoid set, and $\Phi$ a correspondence from $X$ to $Y$. Then the following are equivalent:

(a) The subsets $\bigcap\left\{\Phi^{*}(y): y \in B\right\}, B \subset Y$, are connected or empty.

(b) For every pair $\left(x_{1}, x_{2}\right) \in X \times X$ there exists a connected set $C \supset\left\{x_{1}, x_{2}\right\}$ such that

$$
\Phi(x) \subset \Phi\left(x_{1}\right) \cup \Phi\left(x_{2}\right) \text { for all } x \in C .
$$

(c) For every pair $\left(x_{1}, x_{2}\right) \in X \times X$ the set $\left\{x \in X: \Phi(x) \subset \Phi\left(x_{1}\right) \cup \Phi\left(x_{2}\right)\right\}$ is connected.

Proof. (a) $\Rightarrow$ (c). For $B=Y-\left(\Phi\left(x_{1}\right) \cup \Phi\left(x_{2}\right)\right)$ we have $\bigcap\left\{\Phi^{*}(y): y \in B\right\}=$ $\left\{x \in X: \Phi(x) \subset \Phi\left(x_{1}\right) \cup \Phi\left(x_{2}\right)\right\}$.

(c) $\Rightarrow$ (b) is obvious.

(b) $\Rightarrow\left(\right.$ a). For $\left\{x_{1}, x_{2}\right\} \subset D:=\bigcap\left\{\Phi^{*}(y): y \in B\right\}, B \subset Y$, choose $C$ according to (b). Then we have $\left\{x_{1}, x_{2}\right\} \subset C \subset D$. Hence $D$ is connected.

Remark 2. Let $X$ and $Y$ be topological spaces and $\Phi$ a correspondence from $X$ to $Y$.

(a) $\Phi$ is upper semicontinuous iff $\bigcap\left\{\Phi^{*}(y): y \in F\right\}$ is open for every closed $F \subset Y$.

(b) $\Phi$ is lower semicontinuous iff $\bigcap\left\{\Phi^{*}(y): y \in G\right\}$ is closed for every open $G \subset Y$.

We can now reformulate Theorems 1 and 2:

Theorem $\mathbf{1}^{*}$. For a correspondence $\Phi$ from $X$ to $Y$ the following are equivalent:

(a) $\Phi$ has a constant selector.

(b) There exist topologies on $X$ and $Y$ such that

(o) $Y$ is compact, 
(i) every value $\Phi(x), x \in X$, is closed,

(ii) $\Phi$ is upper semicontinuous,

(iii) every subset $\bigcap\{\Phi(x): x \in A\}, A \in \mathscr{E}(X)$, is connected or empty,

(iv) every subset $\bigcap\left\{\Phi^{*}(y): y \in B\right\}, B \subset Y$, is connected or empty.

Proof. (a) $\Rightarrow$ (b). Choose $\hat{y} \in \bigcap\{\Phi(x): x \in X\}$. Then for the topologies $\mathscr{T}_{X}=\{\varnothing, X\}$ and $\mathscr{T}_{Y}=\{G \subset Y: \hat{y} \notin G\} \cup\{Y\}$ conditions (o)-(iv) are satisfied.

(o) and (iv) are obvious.

(ii) For $G \in \mathscr{T}_{Y}$ and $U=\{x \in X: \Phi(x) \subset G\}$ we have

$$
U \neq \varnothing \Rightarrow \hat{y} \in G \Rightarrow G=Y \Rightarrow U=X .
$$

(i), (iii) Every subset of $Y$ that contains $\hat{y}$ is closed and connected.

(b) $\Rightarrow(a)$. Suppose that (a) is violated. Then by conditions (o) and (i) there is an $A \in \mathscr{E}(X)$ such that

$$
\bigcap\{\Phi(x): x \in A\}=\varnothing .
$$

Step 1. We may choose $A$ such that

$$
\bigcap\{\Phi(x): x \in C\} \neq \varnothing \text { for all } C \subset X \text { with } \operatorname{card} C<\operatorname{card} A .
$$

Since $\Phi(x)$ is nonvoid for every $x \in X$, there exist $x_{1}, x_{2} \in A$ with $x_{1} \neq x_{2}$. We set $E=A-\left\{x_{1}, x_{2}\right\}$ and $\Psi(x)=\bigcap\{\Phi(t): t \in E \cup\{x\}\}$. Then by (2) and (iii)

$$
\text { every } \Psi(x), x \in X \text {, is nonvoid, closed, and connected . }
$$

For $Z:=\left\{x \in X: \Psi(x) \subset \Phi\left(x_{1}\right) \cup \Phi\left(x_{2}\right)\right\}$ and

$$
M_{i}:=\left\{x \in Z: \Psi(x) \subset \Phi\left(x_{i}\right)\right\}, \quad i \in\{1,2\},
$$

we have $(\alpha) x_{i} \in M_{i}, i \in\{1,2\},(\beta) \quad M_{1} \cap M_{2}=\varnothing$, and $(\gamma) \quad M_{1} \cup M_{2}=Z$. Here $(\alpha)$ is obvious and $(\beta),(\gamma)$ follow from (1) and (3). (Observe that for $x \in Z$ we have $\Psi(x) \subset \Psi\left(x_{1}\right) \cup \Psi\left(x_{2}\right)$ and $\Psi\left(x_{1}\right) \cap \Psi\left(x_{2}\right)=\varnothing$ by (1).

Step 2. We now show that $M_{1}$ and $M_{2}$ are both open in $Z$. To see this, let $\bar{x} \in M_{i}$ for $i \in\{1,2\}$. Then $G:=X-\Psi\left(x_{3-i}\right)$ is open and (1) implies $\Psi(\bar{x}) \subset \Psi\left(x_{i}\right) \subset G$, hence $\Phi(\bar{x}) \subset G$. From (ii) it follows that $U=\{x \in X$ : $\Phi(x) \subset G\}$ is an open neighborhood of $\bar{x}$. Now for $V=U \cap Z$ we infer from $(\gamma)$

$$
x \in V \Rightarrow \varnothing=\Phi(x) \cap \Psi\left(x_{3-i}\right)=\Psi(x) \cap \Phi\left(x_{3-i}\right) \Rightarrow x \in M_{i} .
$$

So $\bar{x} \in V \subset M_{i}$, i.e., $M_{i}$ is open in $Z$.

Step 3. From the identity $Z=\bigcap\left\{\Phi^{*}(y): y \in B\right\}$ for $B=(\bigcap\{\Phi(t): t \in E\})$ $-\left(\Phi\left(x_{1}\right) \cup \Phi\left(x_{2}\right)\right)$, it follows by (iv) that $Z$ is connected. Now $(\alpha),(\beta)$, and $(\gamma)$ together with Step 2 lead to a contradiction.

Corollary 1. Let $X$ and $Y$ be topological spaces and $\Phi$ a correspondence from $X$ to $Y$ such that

(i) $Y$ is compact,

(ii) $\mathrm{Gr} \Phi$ is closed (in the product topology),

(iii) every subset $\bigcap\{\Phi(x): x \in A\}, A \in \mathscr{E}(X)$, is connected or empty, 
(iv) for every pair $\left(x_{1}, x_{2}\right) \in X \times X$ there is a connected set $C \supset\left\{x_{1}, x_{2}\right\}$ such that

$$
\Phi(x) \subset \Phi\left(x_{1}\right) \cup \Phi\left(x_{2}\right) \quad \text { for all } x \in C .
$$

Then $\Phi$ has a constant selector.

Proof. It is well known $[9,7.1 .16]$ that (i) and (ii) imply conditions (i) and (ii) in Theorem $1^{*}$. Together with Remark 1 the assertion follows.

Remark 3. (a) The proof of Theorem $1^{*}$ shows that conditions (b)(i)-(iv) alone imply

$$
\bigcap\{\Phi(x): x \in A\} \neq \varnothing \quad \text { for all } A \in \mathscr{E}(X) .
$$

Therefore, Theorem $1^{*}$ remains true if condition (o) is replaced by

$(0)^{*} \Phi\left(x_{0}\right)$ is compact for some $x_{0} \in X$.

(b) Similarly, Corollary 1 remains true if condition (i) is replaced by

(i) * every value $\Phi(x), x \in X$, is compact.

(To see this, proceed as in the proof of Theorem $1^{*}$. Observe that in Step 2 the set $X-G=\Psi\left(x_{3-i}\right)$ is closed and compact, so as in the proof of Theorem 7.1.16 in [9] it follows that $U$ is open.) This is Stachó's Proposition 2 in [16]. Corollary 1 is also an immediate consequence of Simon's Theorem 8 in [14]. (Compare Remark 5(ii) below.) A recent paper of Ch. D. Horvath $(A$ connectivity approach to minimax inequalities, preprint, 1991) contains further work of this type.

(c) Finally, Theorem $1^{*}$ remains true if condition (iii) is assumed for all $A \subset X$, but it fails if condition (iv) is only assumed for all $B \in \mathscr{E}(Y)$ (compare Example 1.1).

Theorem 2* For a correspondence $\Phi$ from $X$ to $Y$ the following are equivalent:

(a) $\bigcap\{\Phi(x): x \in A\} \neq \varnothing$ for all $A \in \mathscr{E}(X)$.

(b) There exist topologies on $X$ and $Y$ such that

(i) every value $\Phi(x), x \in X$, is open,

(ii) $\Phi$ is lower semicontinuous,

(iii) every subset $\bigcap\{\Phi(x): x \in A\}, A \in \mathscr{E}(X)$, is connected or empty,

(iv) every subset $\bigcap\left\{\Phi^{*}(y): y \in B\right\}, B \subset Y$, is connected or empty.

Proof. (a) $\Rightarrow$ (b). Take the topologies $\mathscr{T}_{X}=\{\varnothing, X\}$, and

$$
\mathscr{T}_{Y}=\{G \subset Y: \exists A \in \mathscr{E}(X) \text { such that } G \supset \bigcap\{\Phi(x): x \in A\}\} \cup\{\varnothing\} .
$$

Then conditions (i), (ii), and (iv) are obviously satisfied. It remains to show that every nonvoid subset $C:=\bigcap\{\Phi(x): x \in A\}, A \in \mathscr{E}(X)$, is $\mathscr{T}_{Y}$-connected. To see this, let $C \subset G_{1} \cup G_{2}$ with $G_{1}, G_{2} \in \mathscr{T}_{Y}-\{\varnothing\}$. Choose $A_{i} \in \mathscr{E}(X)$ with $G_{i} \supset \bigcap\left\{\Phi(x): x \in A_{i}\right\}, i \in\{1,2\}$. Then $C \cap G_{1} \cap G_{2} \supset \bigcap\{\Phi(x): x \in$ $\left.A \cup A_{1} \cup A_{2}\right\} \neq \varnothing$.

(b) $\Rightarrow$ (a). Suppose that there is a set $A \in \mathscr{E}(X)$ with $\bigcap\{\Phi(x): x \in A\}=\varnothing$. We proceed as in the proof of Theorem $1^{*}$. Here the sets $\Psi(x), x \in X$, are open and the proof of Step 2 has to be modified as follows: For $i \in\{1,2\}$ let $\bar{x} \in M_{i}$. Then $G=\Psi\left(x_{i}\right)$ is open, and from $\Phi(\bar{x}) \cap G=\Psi(\bar{x}) \cap \Phi\left(x_{i}\right)=$ $\Psi(\bar{x}) \neq \varnothing$ and (ii), we conclude that $U:=\{x \in X: \Phi(x) \cap G \neq \varnothing\}$ is an open neighborhood of $\bar{x}$. For $V=U \cap Z$ we infer from $(\gamma)$

$$
x \in V \Rightarrow \Phi(x) \cap \Psi\left(x_{i}\right) \neq \varnothing \Rightarrow \Psi(x) \cap \Phi\left(x_{i}\right) \neq \varnothing \Rightarrow x \notin M_{3-i} \Rightarrow x \in M_{i} .
$$


Together with Remark 2 we obtain

Corollary 2. Let $X$ and $Y$ be topological spaces and $\Phi$ a correspondence from $X$ to $Y$ such that

(i) every $\Phi(x), x \in X$, is open,

(ii) every $\Phi^{*}(y), y \in Y$, is closed,

(iii) every subset $\bigcap\{\Phi(x): x \in A\}, A \in \mathscr{E}(X)$, is connected or empty,

(iv) every subset $\bigcap\left\{\Phi^{*}(y): y \in B\right\}, B \subset Y$, is connected or empty.

Then $\bigcap\{\Phi(x): x \in A\}$ is nonvoid for every $A \in \mathscr{E}(X)$.

Example 1. Let $X=Y=\mathbb{N}$ be endowed with the "cofinite topology" $\mathscr{T}=$ $\{G \subset \mathbb{N}: \mathbb{N}-G \in \mathscr{E}(\mathbb{N})\} \cup\{\varnothing, \mathbb{N}\}$.

Example 1.1. Let $C_{x}=\{x\}, x \in \mathbb{N}$. Here conditions (b)(o)-(iii) of Theorem 1 are satisfied, and condition (b)(iv) holds for all $B \in \mathscr{E}(Y) \cup\{\varnothing, \mathbb{N}\}$. In particular, Theorem 1 fails if condition (b)(iv) is only assumed for all closed $B \subset Y($ as in (b)(ii)).

Example 1.2. Let $C_{x}=\Phi(x)=\mathbb{N}-\{x\}$. Here all assumptions of Theorem 2(b) and of Corollary 2 are fulfilled.

Example 2. Let $X=Y=\{1,2\}$ be endowed with the discrete topology and take again $C_{x}=\{x\}, x \in X$. Here all conditions of Theorem 2(b) are satisfied with the only exception that condition (iv) does not hold for $B=\varnothing$. Of course, $C_{1} \cap C_{2}=\varnothing$.

Example 3. Let $X=\{1,2\}, Y=\{1,2,3\}, \Phi(1)=\{1,2\}$, and $\Phi(2)=$ $\{2,3\}$. Let $\mathscr{T}_{X}$ be a topology on $X$ such that the sets $\Phi^{*}(1)=\{2\}$ and $\Phi^{*}(3)=\{1\}$ are both open (resp. both closed). Then $\mathscr{T}_{X}$ is the discrete topology, and $X=\bigcap\left\{\Phi^{*}(y): y \in \varnothing\right\}$ is not connected. Hence, conditions (ii) and (iv) of Corollary 1 or 2 cannot both be satisfied. On the other hand, conditions (b)(o)-(iv) of Theorem $1^{*}$ are fulfilled if we take the topologies $\mathscr{T}_{X}=\{\varnothing, X\}$ and $\mathscr{T}_{Y}=\{G \subset Y: 2 \notin G\} \cup\{Y\}=\{\varnothing,\{1\},\{3\},\{1,3\}, Y\}$. This example shows that the results analogous to the converses of Theorems $1^{*}$ and $2^{*}$ do not hold in the situation of Corollaries 1 and 2 and that Theorem $1^{*}$ cannot be derived from Stachó's Proposition 2 in [16].

Remark 4. If $\operatorname{Gr} \Phi$ is open then, of course, conditions (i) and (ii) of Corollary 2 are satisfied, but not conversely as Example 1.2 shows. It is an open problem whether Corollary 1 remains true if, instead of a closed graph, one only assumes that all $\Phi(x), x \in X$, are closed and all $\Phi^{*}(y), y \in Y$, are open. Compare also [8, (4.4), (4.5); 7, (Remark); 13].

\section{MINIMAX AND PREMINIMAX VERSIONS}

For the rest of the paper let $X$ and $Y$ be nonvoid sets and $a: X \times Y \rightarrow \overline{\mathbb{R}}$ an extended real-valued function on the cartesian product. We set

$$
\begin{gathered}
a_{*}=\sup _{x \in X} \inf _{y \in Y} a(x, y), \quad a^{*}=\inf _{y \in Y} \sup _{x \in X} a(x, y), \\
\tilde{a}^{*}=\sup _{A \in \mathscr{E}(X)} \inf _{y \in Y} \max _{x \in A} a(x, y) .
\end{gathered}
$$

Then $a_{*} \leq \tilde{a}^{*} \leq a^{*}$ is always true. We say that $a$ fulfills 
(the minimax relation) MM iff $a_{*}=a^{*}$,

(the preminimax relation) PMM iff $\tilde{a}^{*}=a_{*}$, and

(the minimum minimax relation) MMM iff $\sup _{x \in X} a(x, \hat{y})=a_{*}$ for some $\hat{y} \in Y$.

The intersection problem and the minimax problem are related as follows:

Remark 5. Let $X$ and $Y$ be nonvoid sets.

(i) A function $a: X \times Y \rightarrow \overline{\mathbb{R}}$ satisfies MMM iff

$$
\Phi(x):=\left\{a(x, \cdot) \leq a_{*}\right\}, \quad x \in X,
$$

is a correspondence from $X$ to $Y$ with constant selector.

(ii) A correspondence $\Phi$ from $X$ to $Y$ has a constant selector iff the function $a$ :

$$
a(x, y):=1_{\Phi^{*}(y)}(x), \quad x \in X, y \in Y,
$$

satisfies MM (hence MMM).

(iii) A function $a: X \times Y \rightarrow \overline{\mathbb{R}}$ satisfies MM iff for every $\lambda>a_{*}$

$$
\Phi(x):=\{a(x, \cdot)<\lambda\}, \quad x \in X,
$$

is a correspondence from $X$ to $Y$ with constant selector.

(iv) A function $a: X \times Y \rightarrow \overline{\mathbb{R}}$ satisfies PMM iff for every $\lambda>a_{*}$

$$
\Phi(x):=\{a(x, \cdot)<\lambda\}, \quad x \in X,
$$

satisfies $\bigcap\{\Phi(x): x \in A\} \neq \varnothing$ for all $A \in \mathscr{E}(X)$.

(v) A correspondence $\Phi$ from $X$ to $Y$ satisfies $\bigcap\{\Phi(x): x \in A\} \neq \varnothing$ for all $A \in \mathscr{E}(X)$ iff the function $a$ :

$$
a(x, y):=1_{\Phi^{*}(y)}(x), \quad x \in X, y \in Y,
$$

satisfies PMM.

Example 4 (Compare [12, Theorem 2; 13, Theorem 1.3]). Let $X$ and $Y$ be topological spaces such that

(o) $Y$ is compact,

(i) $a$ is lower semicontinuous (in the product topology),

(ii) all subsets $\bigcap\left\{\left\{a(x, \cdot) \leq a_{*}\right\}: x \in A\right\}, A \in \mathscr{E}(X)$, are connected or empty,

(iii) all subsets $\bigcap\left\{\left\{a(\cdot, y)>a_{*}\right\}: y \in B\right\}, B \subset Y$, are connected or empty. Then MMM holds.

Proof. Apply Corollary 1 and Remarks 1 and 5(i).

In the following, if $X$ and $Y$ are topological spaces then we say that $a$ is upper connected in $x$ iff the sets

$$
\left\langle x_{1}, x_{2}\right\rangle_{X}:=\left\{x \in X: a(x, \cdot) \geq a\left(x_{1}, \cdot\right) \wedge a\left(x_{2}, \cdot\right)\right\}, \quad\left(x_{1}, x_{2}\right) \in X \times X,
$$

are connected and that $a$ is lower connected in $y$ iff the sets

$$
\left\langle y_{1}, y_{2}\right\rangle_{Y}:=\left\{y \in Y: a(\cdot, y) \leq a\left(\cdot, y_{1}\right) \vee a\left(\cdot, y_{2}\right)\right\}, \quad\left(y_{1}, y_{2}\right) \in Y \times Y,
$$

are connected. 
Example 4.1. Suppose that there are topologies on $X$ and $Y$ such that

(o) $Y$ is compact,

(i) $a$ is lower semicontinuous,

(ii) $a$ is upper connected in $x$,

(iii) $a$ is lower connected in $y$.

Then MMM holds.

Proof. By Remark 1, applied to $\Phi(x)=\left\{a(x, \cdot) \leq a_{*}\right\}$, condition (ii) implies condition (iii) in Example 4. Similarly, (iii) implies condition (ii) in Example 4 because $\left\{y_{1}, y_{2}\right\} \subset D:=\bigcap\left\{\left\{a(x, \cdot) \leq a_{*}\right\}: x \in A\right\}, A \in \mathscr{E}(X)$, implies $\left\langle y_{1}, y_{2}\right\rangle_{Y} \subset D$.

Example 4.2. Let $X$ and $Y$ be convex subsets of two (Hausdorff) topological vector spaces.

(i) If $a$ is quasiconcave in $x$ then $a$ is upper connected in $x$.

(ii) If $a$ is quasiconvex in $y$ then $a$ is lower connected in $y$.

In connection with Example 4.1 this leads to Ha's minimax theorem [4, Theorem 4].

Example 5. Let $X$ and $Y$ be topological spaces such that

(i) $a$ is upper semicontinuous in $x$,

(ii) $a$ is upper semicontinuous in $y$,

(iii) $a$ is upper connected in $x$,

(iv) $a$ is lower connected in $y$.

Then PMM holds.

Proof. For $\lambda>a_{*}$ let $\Phi(x)=\{a(x, \cdot)<\lambda\}, x \in X$. Then conditions (i) and (ii) imply conditions (i) and (ii) in Corollary 2 , and conditions (iii) and (iv) imply conditions (iv) and (iii) in Corollary 2 as in the proof of Example 4.1. Now by Remark 5(iv) the assertion follows from Corollary 2.

As a special case we obtain the following unconventional version of Sion's minimax theorem [15].

Example 5.1. Let $X$ and $Y$ be convex subsets of topological vector spaces such that

(i) $a$ is upper semicontinuous in $x$,

(ii) $a$ is upper semicontinuous in $y$,

(iii) $a$ is quasi-concave in $x$,

(iv) $a$ is quasi-convex in $y$.

Assume, moreover, that there is another topology on $Y$ such that

(o) $Y$ is compact and $a$ is lower semicontinuous in $y$.

Then MMM holds.

Proof. By Examples 4.2 and 5 conditions (i)-(iv) imply $a_{*}=\tilde{a}^{*}$, and from (o) we easily infer $\tilde{a}^{*}=a^{*}$.

\section{AN OPEN PROBLEM}

In $[1,2]$ Fan proved the following theorem with the aid of his infinitedimensional version of the Knaster-Kuratowski-Mazurkiewicz Theorem: 
Theorem (Fan). Let $X$ be a compact convex subset of a topological vector space. Assume that $\Phi$ is a correspondence from $X$ to $X$ such that

(i) every $\Phi(x), x \in X$, is closed,

(ii) every $\Phi^{*}(y), y \in X$, is convex,

(iii) $x \in \Phi(x)$ for every $x \in X$.

Then $\Phi$ has a constant selector.

As Fan's Theorem has a lot of interesting applications it would be very desirable to have a simple proof - and perhaps even a generalization to nonconvex situations-of this important result.

Problem. In the above situation let $\mathscr{T}_{1}$ be the (relative) vector space topology on $X$. Find a topology $\mathscr{T}_{2}$ on $X$ such that $\Phi$ is an upper (resp. lower) semicontinuous correspondence from $\left(X, \mathscr{T}_{1}\right)$ to $\left(X, \mathscr{T}_{2}\right)$, every value $\Phi(x)$, $x \in X$, is $\mathscr{T}_{2}$-closed (resp. $\mathscr{T}_{2}$-open), and every subset $\bigcap\{\Phi(x): x \in A\}$, $A \in \mathscr{E}(X)$, is $\mathscr{T}_{2}$-connected or empty.

If this problem is solved then Fan's Theorem is an immediate consequence of Theorem $1^{*}$ and Remark 3 or Theorem $2^{*}$, respectively. On the other hand, once Fan's Theorem is proved such topologies can be found easily: Take $\mathscr{T}_{2}=\mathscr{T}_{Y}$ as in the proof of Theorem $1^{*}\left(2^{*}\right)$.

Question. Can the above problem be solved without using any version of the KKM-Theorem?

\section{ACKNOWLEDGMENTS}

Special thanks are due to Stephen Simons whose talk in spring 1990 in Oberwolfach on a preliminary version of [14] and the ensuing discussion gave an impetus for the present investigations, to Heinz König whose recent paper [13] and the discussion about it have influenced the final exposition of this paper, and to the referee whose helpful comments led to an improvement of the presentation.

\section{REFERENCES}

1. K. Fan, A generalization of Tychonoff's fixed point theorem, Math. Ann. 142 (1961), 305310.

2. __ A minimax inequality and applications, Inequalities. III, Academic Press, New York and London, 1972, pp. 103-113.

3. M. A. Geraghty and B.-L. Lin, Topological minimax theorems, Proc. Amer. Math. Soc. 91 (1984), 377-380.

4. Ch.-W. Ha, Minimax and fixed point theorems, Math. Ann. 248 (1980), 73-77.

5. Ch. Horvath, Quelques théorèmes en théorie des Mini-Max, C. R. Acad. Sci. Paris Ser. I 310 (1990), 269-272.

6. I. Joó, A simple proof for von Neumann's minimax theorem, Acta Sci. Math. 42 (1980), 91-94.

7. __ On some convexities, Acta Math. Hungar. 54 (1989), 163-172.

8. J. Kindler and R. Trost, Minimax theorems for interval spaces, Acta Math. Hungar $\mathbf{5 4}$ (1989), 39-49.

9. E. Klein and A. C. Thomson, Theory of correspondences, Wiley, New York, 1984.

10. H. Komiya, Elementary proof for Sion's minimax theorem, Kodai Math. J. 11 (1988), 5-7. 
11. On minimax theorems without linear structure, Hiyoshi Review of Natural Science 8 (1990), 74-78.

12. V. Komornik, Minimax theorems for upper semicontinuous functions, Acta Math. Acad. Sci. Hungar. 40 (1982), 159-163.

13. H. König, A general minimax theorem based on connectedness, Arch. Math. (to appear).

14. S. Simons, A flexible minimax theorem, Acta Math. Hungar. (to appear).

15. M. Sion, On general minimax theorems, Pacific J. Math. 8 (1958), 171-176.

16. L. L. Stachó, Minimax theorems beyond topological vector spaces, Acta Sci. Math. 42 (1980), 157-164.

17. H. Tuy, On a general minimax theorem, Soviet Math. Dokl. 15 (1974), 1689-1693.

18. Wu Wen-Tsün, A remark on the fundamental theorem in the theory of games, Sci. Rec. (N.S.) 3 (1959), 229-233.

Technische Hochschule, Fachbereich Mathematik, Schlossgartenstrasse 7, D-6100 DARMSTADT, GermanY 